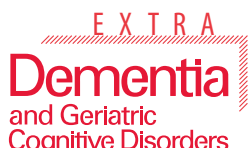

Cognitive Disorders

Dement Geriatr Cogn Disord Extra 2019;9:250-259

\title{
Association between Chronic Obstructive Pulmonary Disease and Dementia: Systematic Review and Meta-Analysis of Cohort Studies
}

\author{
Ying Wang ${ }^{a}$ Xiaotong $\mathrm{Li}^{\mathrm{a}}$ Biying Wei ${ }^{\mathrm{a}}$ Tao-Hsin Tung ${ }^{\mathrm{b}, \mathrm{c}}$ Ping Tao ${ }^{\mathrm{d}, \mathrm{e}}$ \\ Ching-Wen Chien ${ }^{a}$ \\ anstitute for Hospital Management, Tsinghua University, Shenzhen Campus, Shenzhen, \\ China; ${ }^{b}$ Faculty of Public Health, College of Medicine, Fu Jen Catholic University, New Taipei, \\ Taiwan; ' Department of Medical Research and Education, Cheng Hsin General Hospital, \\ Taipei, Taiwan; ${ }^{\mathrm{d}}$ Department of Medical Affair and Planning, Taipei Veterans General \\ Hospital, Taipei, Taiwan; eInstitute of Health Policy and Management, National Taiwan \\ University, Taipei, Taiwan
}

\section{Keywords}

Chronic obstructive pulmonary disease $\cdot$ Dementia $\cdot$ Meta-analysis

\section{Abstract}

Background: Chronic obstructive pulmonary disease (COPD) is a common disease among the elderly, which has been linked to cognitive decline. However, the relationship between COPD and dementia remains unclear. Summary: We conducted a systematic literature review by searching databases such as Pubmed, Embase, EBSCO, and Cochrane Library (from inception to April 18, 2018) for studies on COPD that also investigated the prevalence of dementia. We found 3 cohort studies including a total of 39,392 COPD patients. Then we applied the Newcastle-Ottawa Scale to evaluate the risk of bias. Key Messages: COPD patients faced a higher risk of dementia (HR 1.46; $95 \% \mathrm{Cl} 1.22-1.75 ; p<0.001)$. Subgroup analysis on gender determined that the association between COPD and dementia was stronger in male patients (HR $1.49,95 \% \mathrm{Cl} 1.20-1.86, p<0.001$ ) than in female patients (HR 1.41, 95\% Cl 1.27-1.57, $p<0.001$ ). A subset study of patients aged $>65$ years revealed that the HR was greater for patients aged $\geq 75$ years (HR 1.46, 95\% Cl 1.07-2.00, $p=0.02$ ) than for those aged $65-74$ years (HR 1.40, 95\% $\mathrm{Cl} 1.28-1.53, p<0.001$ ). The cohort studies included were from similar population-based databases, suggesting possible regional limitations and publication bias. 
Wang et al.: Association between Chronic Obstructive Pulmonary Disease and Dementia

\section{Introduction}

Dementia is the fourth leading cause of death among the elderly, following cancer, heart disease, and cerebrovascular disease [1]. Dementia-related mortality rates have been reported to range from 0.8 to $27 \%$ worldwide [2]. Dementia is a progressive neurocognitive disease. In the absence of any disease-modifying treatment, increasing focus has been placed on primary prevention (to reduce the risk of development) and early intervention (to slow progression) therapies. A better understanding of the risk factors of dementia is crucial to improving therapeutic interventions. In addition to a number of well-described cardiovascular risk factors, there is a growing body of evidence which suggests a link between COPD and the development of dementia. The first report on cognitive impairment in COPD patients was published in 1982 [3]. Since that time, numerous studies have documented a relationship between COPD and cognitive and psychological dysfunctions [4-6]. Dementia has also been associated with age, obesity, genetics, education, smoking, alcohol, cerebral trauma, mid-life hypertension, stroke, diabetes, hyperlipidemia, myocardial infarction, and heart failure [7-11].

Very little previous research has investigated a direct relationship between COPD and dementia. Furthermore, to the best of our knowledge, no previous study has determined whether COPD represents prodromal symptoms or acts as an independent risk factor of dementia among the elderly. In this work, we conducted a meta-analysis of previous studies with the primary objective of assessing the risk of dementia in patients with COPD, which we believe is the most salient aspect of current research. We also sought to determine whether further studies on the potential link between COPD and dementia are warranted and to provide guidance if that is the case.

\section{Materials and Methods}

\section{Search Strategy}

A thorough search was conducted on the PubMed, Web of Science, EBSCO, Embase, Cochrane Library, CNKI (China National Knowledge Infrastructure), and WanFang databases using the following keywords: "(Chronic Obstructive Pulmonary Disease OR COPD OR Chronic Obstructive Airway Disease OR COAD OR Chronic Airflow Obstructions OR Chronic Obstructive Lung Disease) AND (Dementia)." No language limitations were imposed, and the search period was from inception of the database to April 18, 2018. An initial screening of studies using the aforementioned keywords was independently performed by 3 researchers (Y. Wang, X. Li, and B. Wei) based on title, abstract, and keywords. In case of disagreement, a fourth person (T.-H. Tung) decided whether to include or exclude the study. For those papers lacking necessary data, attempts were made to contact the original authors. In the event that information on the prevalence of dementia was unavailable, the study was excluded.

\section{Study Selection}

The inclusion criteria for studies was as follows. (1) They employed a cohort study design. (2) They adopted a defined outcome of dementia, as reported by physicians using International Classification of Diseases (ICD) codes. (3) They compared an exposure group that comprised individuals who were clinically diagnosed with COPD and who were followed until a diagnosis of dementia was made, death, or the end of the study period with a control group that comprised subjects without a history of COPD or dementia. (4) They reported results which included a hazard ratio (HR) with a 95\% confidence interval (CI) or raw data which was available for conversion. Cross-sectional or case-control studies that could not be used 


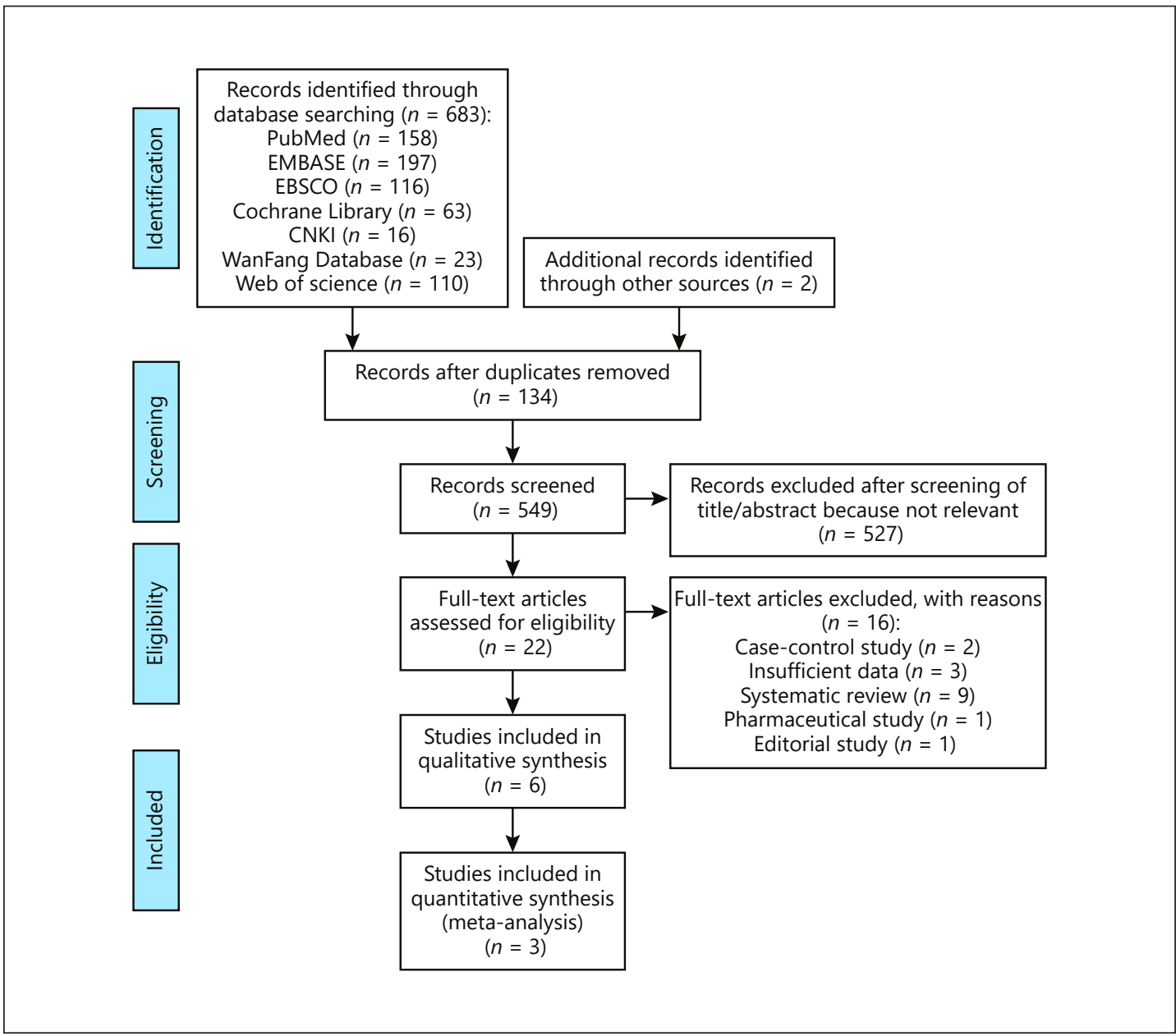

Fig. 1. Flowchart of the article selection. CNKI, China National Knowledge Infrastructure.

to assess the causal relationship between COPD and dementia were excluded. We also excluded conference abstracts, editorials, and systematic reviews that provided limited information. We reviewed the full text of all articles which appeared to be related to COPD and dementia.

\section{Data Extraction}

The following data were extracted from the included studies: first author, year of publication, region, database, study duration, research design, inclusion criteria, type of participants, comparisons, outcomes, and risk estimates. We also adopted the Newcastle-Ottawa Scale, which considers the selection of study groups, comparability, and outcome assessment in evaluating the quality of cohort studies.

\section{Statistical Methods}

Review Manager 5.3 was used to perform the meta-analysis. The risk of outcome was presented as an HR with a 95\% CI. We also used inconsistency statistics. The statistic describes the percentage of variation across studies due to heterogeneity rather than chance alone. A value $\geq 50 \%$ represents substantial heterogeneity. The current meta-analysis was based on a random-effects model due to the expectation of considerable clinical heterogeneity. 
Wang et al.: Association between Chronic Obstructive Pulmonary Disease and Dementia

Subgroup analysis was subsequently employed to analyze associations between dementia and COPD in various groups of people. We divided the studies into a male and female group by origins, and into a group of $<65$-yearolds, 65 - to 74 -year-olds, and $\geq 75$-year-olds by origins. The third study was only excluded from the age-related subgroup analysis due to the unclear classification of age.

\section{Results}

\section{Description of Studies Selected for Inclusion}

A total of 683 studies were identified in the combined search from which we removed 134 duplicate studies. After reviewing the titles and abstracts, a further 527 studies were excluded. This left 22 eligible studies, of which 19 were selected for a full review. All of the reviewed articles were full-text publications [6, 12-29]. 16 articles were excluded for the following reasons: they employed a case-control study design $[28,29]$; they contained insufficient data [20-22]; they involved a systematic review [6,12-19]; they comprised a pharmaceutical study [23]; or they had been published as an editorial [24] (Fig. 1).

After excluding unrelated studies and studies that did not meet our inclusion criteria, 3 prospective cohort studies were available for our meta-analysis. Key details of these studies are summarized in Table 1, including diagnostic criteria and characteristics of exposure groups and control groups. The 3 cohort studies measured the rate of dementia among individuals with COPD during the tracking period. All studies were conducted in Taiwan and were written in English [25-27].

All 3 of the cohort studies used an exposure group in the National Health Insurance Research Database; however, they varied in terms of the period in which diagnoses were made [25-27]. None of the participants included in control groups had a history of COPD. Two of the studies only included patients over 40 years old [25, 27], whereas the third study included patients over 20 years old [26]. The same outcome measures (i.e., diagnoses made in accordance with ICD-9-CM codes) were employed by all 3 studies.

\section{Methodologic Quality of Included Reviews}

One study achieved a moderate Newcastle-Ottawa Scale quality score of 7, and two achieved high scores of 8 , as shown in Table 2.

\section{Association between COPD and Dementia}

All 3 of the studies included in this meta-analysis reported a significant association between COPD and dementia. The first study, by Yeh et al. [25], included 10,260 patients with COPD and asthma and 20,513 control subjects. In that research, the COPD and asthma group had a higher risk of neurodegenerative diseases, wherein the risk of dementia was $1.43 \times$ higher than that of non-COPD patients (HR 1.43, 95\% CI 1.29-1.59). The second study, by Liao et al. [26], followed up 8,640 COPD patients and 17,280 control subjects and reported that COPD increased the risk of dementia (Alzheimer's disease or Parkinson's disease) (HR 1.74, 95\% CI 1.55-1.95). The third study was conducted by Liao et al. [27] and included 20,492 COPD patients and 40,765 control subjects. In that research, COPD was found to be associated with a higher risk of dementia after comorbidities were adjusted for (HR 1.27, 95\% CI 1.201.34). Moreover, the association was stronger among patients who experienced a higher frequency of acute COPD exacerbation events.

Significant associations were shown in Figure 2. The funnel plot in Figure 3 gave no indication of serious publication bias. 
Dementia

and Geriatric

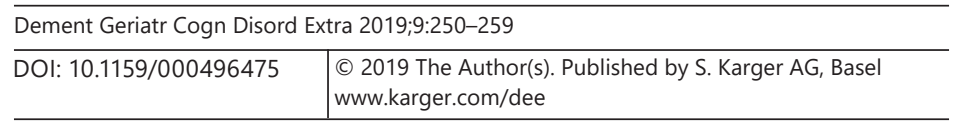

Wang et al.: Association between Chronic Obstructive Pulmonary Disease and Dementia
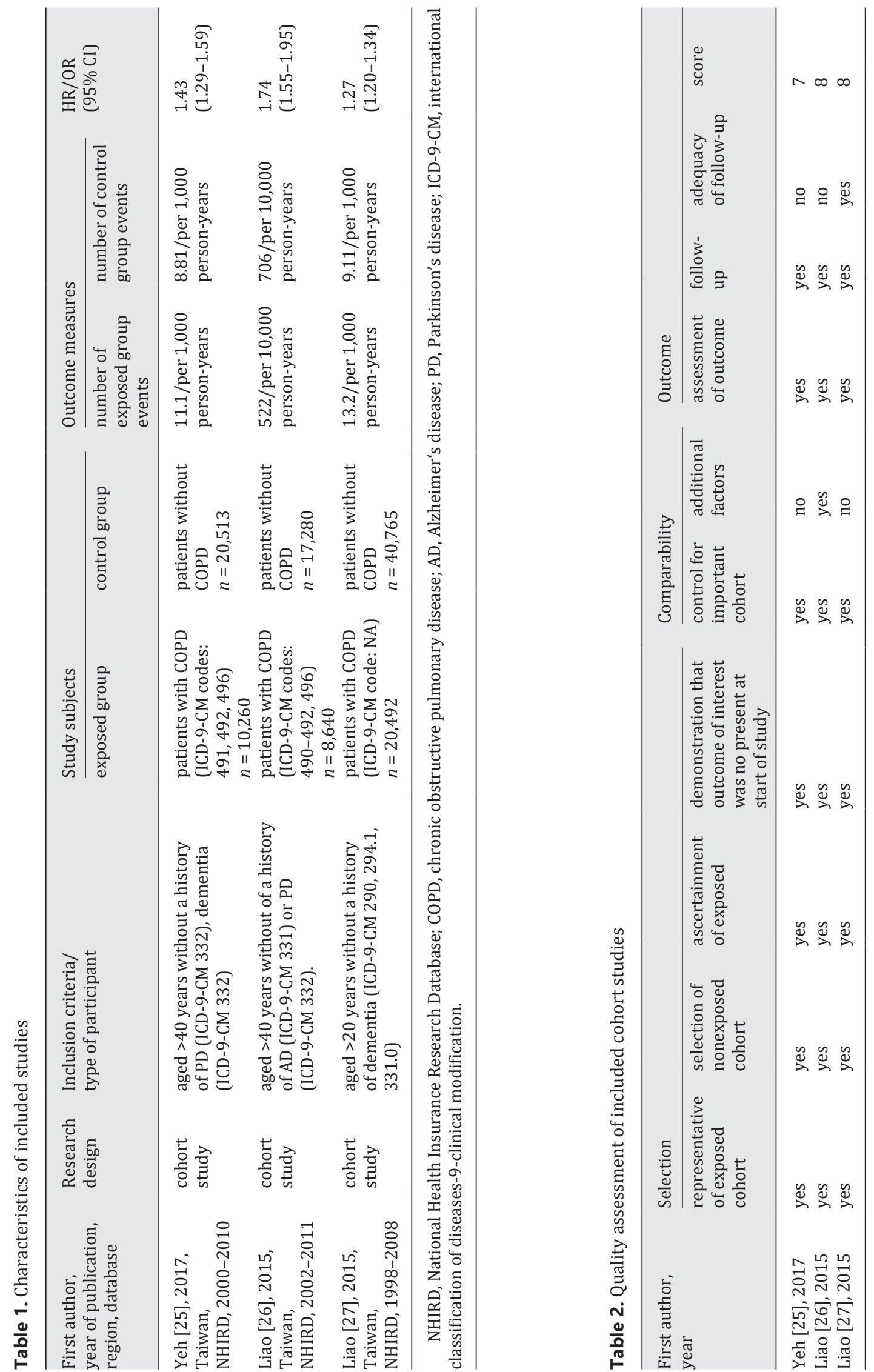
Wang et al.: Association between Chronic Obstructive Pulmonary Disease and Dementia

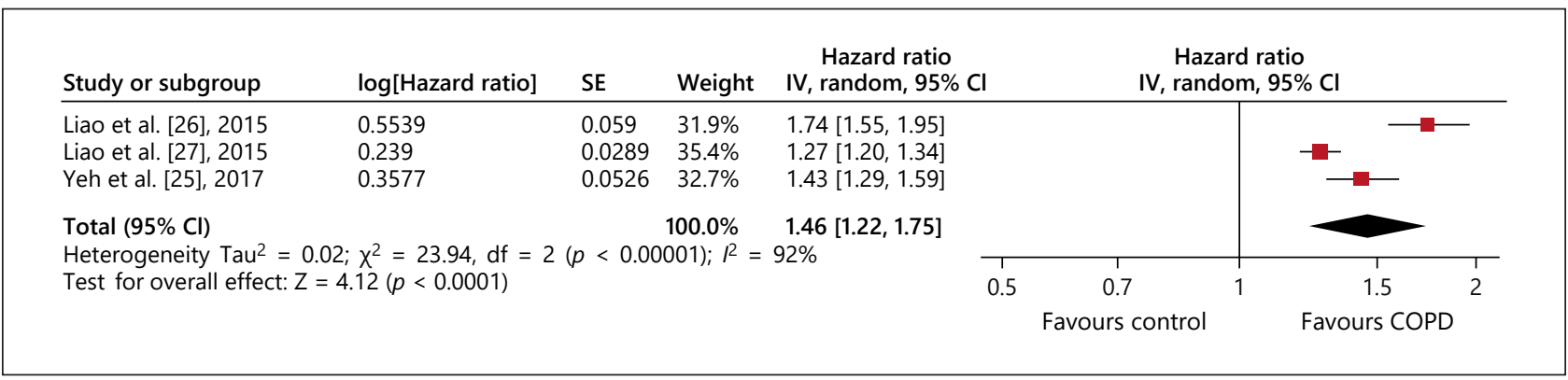

Fig. 2. Meta-analysis of the risk of dementia in all people with COPD. CI, confidence interval; SE, standard error.

Fig. 3. Funnel plot of publication bias of the cohort study.

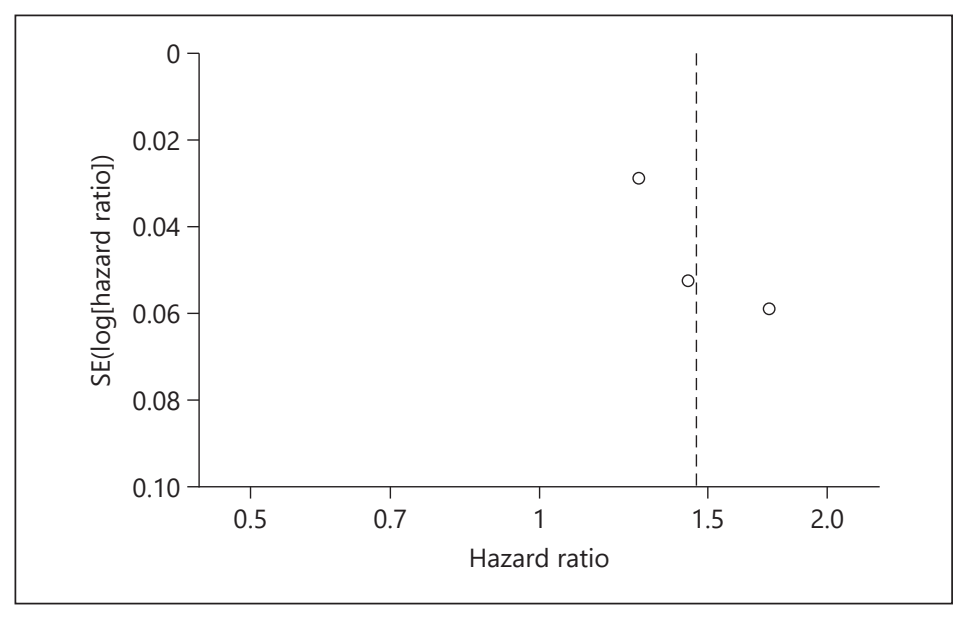

Considerable heterogeneity was observed across studies ( $p<0.001,92 \%)$, indicating a very high degree of variation. Meta-analysis using a random-effects model revealed an increased risk of dementia among patients with COPD (HR 1.46, 95\% CI 1.22-1.75).

To identify which factors were primarily responsible for the heterogeneity we observed, we also conducted subgroup analysis according to gender and age. Subgroup analysis by gender indicated that COPD was significantly associated with dementia (HR 1.49, 95\% CI $1.20-1.86, p<0.001$ ) among male patients. Similar results were obtained for female patients (HR 1.41, 95\% CI 1.27-1.57, $p<0.001$ ) (Fig. 4). Among patients aged $<65$ years, no significant correlation was observed between COPD and dementia (HR 1.80, 95\% CI 1.00-3.22, $p=0.05$ ); however, COPD was significantly associated with dementia in older patients. The HR for patients aged 65-74 years was 1.40 (95\% CI 1.28-1.53, $p<0.001)$, and the HR for patients aged $\geq 75$ years was 1.46 (95\% CI 1.07-2.00, $p=0.02$ ) (Fig. 5).

\section{Discussion}

To the best of our knowledge, this is the first systematic review and meta-analysis to examine associations between COPD and dementia. Our results indicate that patients with COPD face an increased risk of dementia.

Subgroup analysis by age revealed a significant correlation between COPD and dementia among patients over 65 years old. COPD was not a risk factor for dementia among patients 
Wang et al.: Association between Chronic Obstructive Pulmonary Disease and Dementia

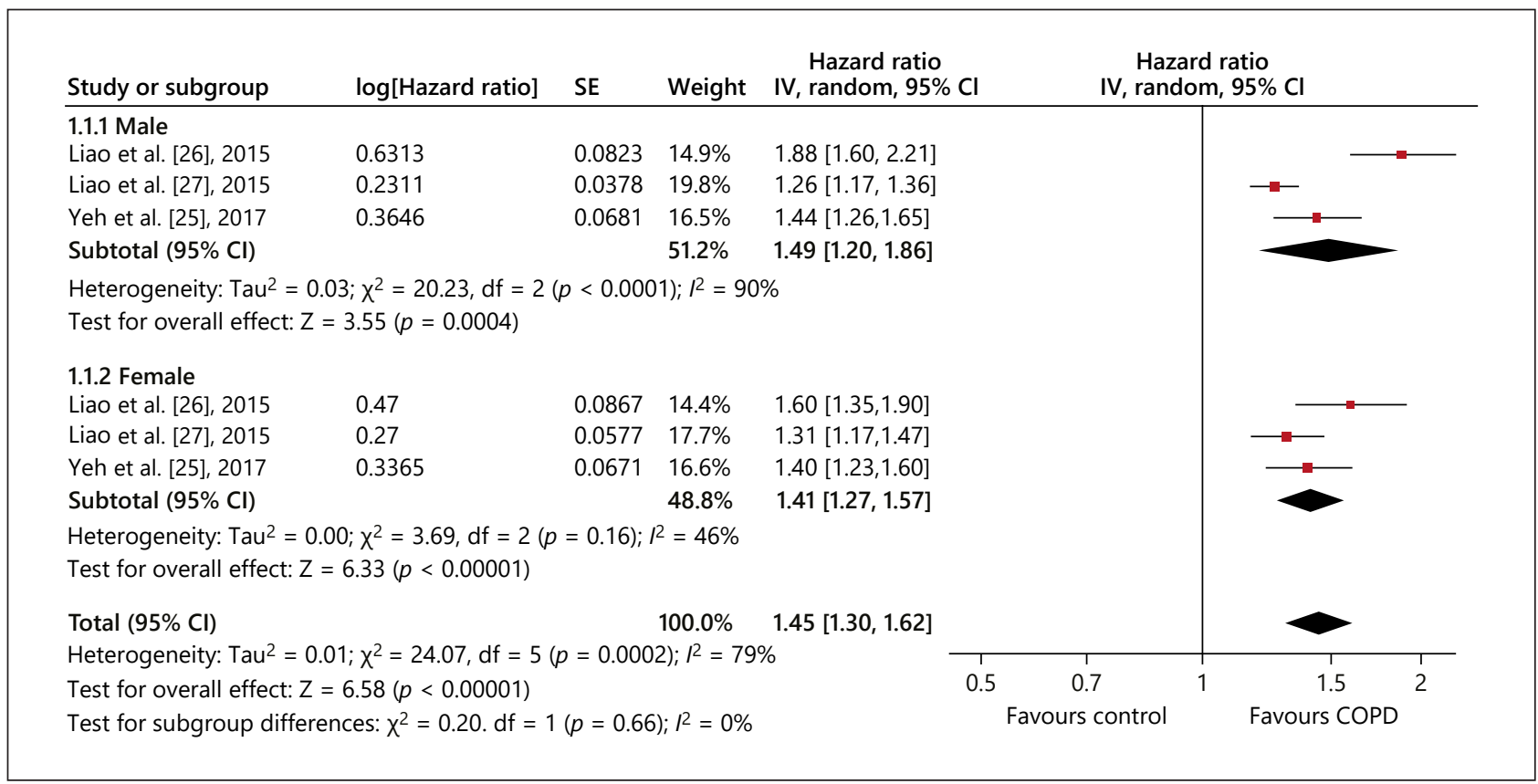

Fig. 4. Subgroup analysis by gender evaluating the risk of dementia in all people with COPD. CI, confidence interval; SE, standard error.

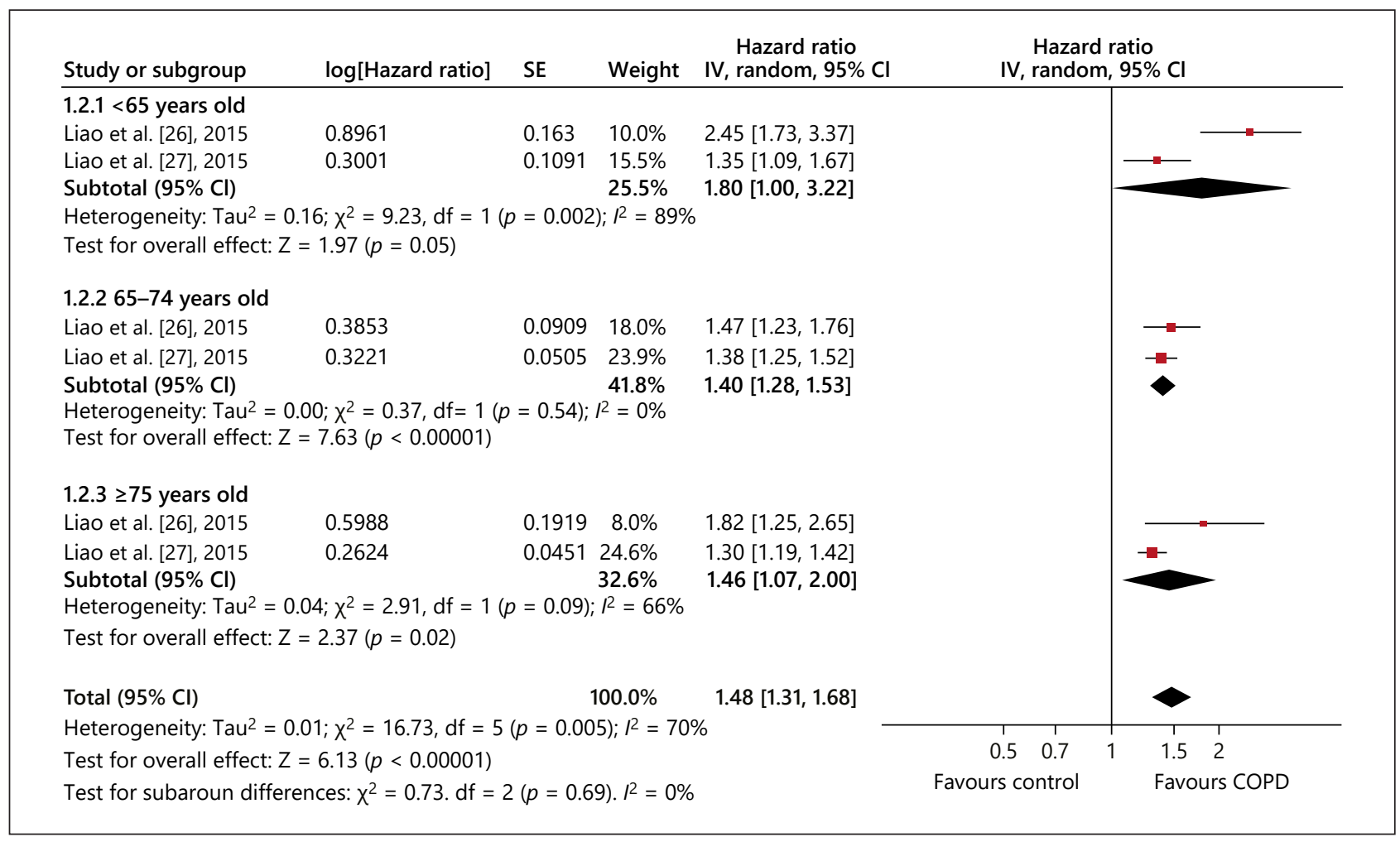

Fig. 5. Subgroup analysis by age group evaluating the risk of dementia in all people with COPD. CI, confidence interval; SE, standard error. 
younger than 65 years old. Subgroup analysis did not reveal a significant difference in the risk of dementia between males and females.

These findings suggest that COPD treatment and prevention of COPD exacerbation are important in reducing the risk of dementia. Nonetheless, additional research will be required to identify the types of treatment which are capable of slowing the progression of dementia among individuals with COPD.

Dementia is a multifactorial disease which has been linked to age, obesity, genetics, education, smoking, alcohol, cerebral trauma, mid-life hypertension, stroke, diabetes, hyperlipidemia, myocardial infarction, and heart failure [7-11]. From a clinical perspective, COPD can lead to hypoxemia and pulmonary encephalopathy, which can have a substantial impact on brain dysfunction [30] and therefore COPD may lead to dementia. COPD has been associated with cognitive decline [13]; however, there does not appear to be any direct evidence of a relationship between COPD and dementia. COPD was first linked to neuropsychological deficit in the early 1980s [31]. A number of researchers have invoked hypoxia to explain the connection between pulmonary failure and brain dysfunction [32]. However, other studies have reported that multiple factors contribute to dementia, including systemic inflammation [30, 33], hypercapnia [34], oxidative stress, and hypoperfusion [35, 36]. Shared risk factors, such as age, smoking, and obesity also contribute, as do commonly associated diseases, such as cerebral disorder, diabetes mellitus, and previous stroke [7-11].

In this study, we addressed the lack of research on the relationship between COPD and dementia with the aim of identifying a causal relationship. We included cohort studies and excluded cross-sectional and case-control studies to increase the strength of the evidence. From a practical perspective, it would not be possible to randomly distribute individuals into the categories "with COPD" and "without COPD;" therefore, randomized controlled trials were excluded from this research. Only cohort studies can be used to detect the effects of long-term COPD (including effects that vary according to disease severity), which may play an important role in the development of dementia.

There were a number of limitations that may have influenced the precision of our metaanalysis. (1) We only identified a few relevant cohort studies, and all of them were conducted in Taiwan. Therefore, there is no way to determine whether the risk of dementia differs among COPD patients in other parts of the world. (2) We were unable to conduct subgroup analysis based on comorbidities or the severity of COPD, due to the fact that the included studies did not provide adequate data for this type of subgroup analysis. This reduced the strength of evidence which indicated an association between COPD and dementia. (3) Diagnostic methods varied among the studies included in our meta-analysis. (4) Variations in matched comorbidities and variables may have introduced bias.

\section{Conclusions}

Results from this meta-analysis revealed that the risk of dementia was higher among patients with COPD than among patients without COPD. However, our research findings could be strengthened through the inclusion of new evidence (e.g., data from different regional areas) to clarify whether a factor such as geographical bias influenced our results. Future meta-analyses which include additional studies could also improve the quality and reliability of findings. 
Wang et al:: Association between Chronic Obstructive Pulmonary Disease and Dementia

\section{Disclosure Statement}

Ying Wang, Xiaotong Li, Biying Wei, Tao-Hsin Tung, Ping Tao, and Ching-Wen Chien declare that they do not have any conflicts of interest.

\section{Funding Sources}

There was no additional financial support from public or private sources.

\section{References}

1 Zhang JT. Pathogenesis and treatment medicine of senile dementia. Herald Med. 2002;21(8):469-71.

2 Ministry of Health China. Annual health statistics, 2003. Beijing: Peking Union Medical University Press; 2003. Available from: http://www.moh.gov.cn/zwgkzt/ptjnj/list.shtml.

3 Grant I, Heaton RK, McSweeny AJ, Adams KM, Timms RM. Neuropsychologic findings in hypoxemic chronic obstructive pulmonary disease. Arch Intern Med. 1982 Aug;142(8):1470-6.

4 Hung WW, Wisnivesky JP, Siu AL, Ross JS. Cognitive decline among patients with chronic obstructive pulmonary disease. Am J Respir Crit Care Med. 2009 Jul;180(2):134-7.

5 Dodd JW, Getov SV, Jones PW. Cognitive function in COPD. Eur Respir J. 2010 Apr;35(4):913-22.

6 Schou L, Østergaard B, Rasmussen LS, Rydahl-Hansen S, Phanareth K. Cognitive dysfunction in patients with chronic obstructive pulmonary disease-a systematic review. Respir Med. 2012 Aug;106(8):1071-81.

7 Cheng G, Huang C, Deng H, Wang H. Diabetes as a risk factor for dementia and mild cognitive impairment: a meta-analysis of longitudinal studies. Intern Med J. 2012 May;42(5):484-91.

$8 \mathrm{Xu} \mathrm{W}$, Tan L, Wang HF, Tan MS, Tan L, Li JQ, et al. Education and risk of dementia: dose-response meta-analysis of prospective cohort studies. Mol Neurobiol. 2016 Jul;53(5):3113-23.

9 Loef M, Walach H. Midlife obesity and dementia: meta-analysis and adjusted forecast of dementia prevalence in the United States and China. Obesity (Silver Spring). 2013 Jan;21(1):E51-5.

10 Mitchell AJ, Beaumont H, Ferguson D, Yadegarfar M, Stubbs B. Risk of dementia and mild cognitive impairment in older people with subjective memory complaints: meta-analysis. Acta Psychiatr Scand. 2014 Dec;130(6): 439-51.

11 Zhong G, Wang Y, Zhang Y, Guo JJ, Zhao Y. Smoking is associated with an increased risk of dementia: a metaanalysis of prospective cohort studies with investigation of potential effect modifiers. PLoS One. 2015 Mar; 10(3):e0118333.

12 Zhang X, Cai X, Shi X, Zheng Z, Zhang A, Guo J, et al. Chronic obstructive pulmonary disease as a risk factor for cognitive dysfunction: a meta-analysis of current studies. J Alzheimers Dis. 2016 Feb;52(1):101-11.

13 Olaithe M, Bucks RS, Hillman DR, Eastwood PR. Cognitive deficits in obstructive sleep apnea: insights from a meta-review and comparison with deficits observed in COPD, insomnia, and sleep deprivation. Sleep Med Rev. 2018 Apr;38:39-49.

14 Slack JA, Giordani B. Domain specific cognitive changes in persons with chronic obstructive pulmonary disease (COPD): a systematic review and meta-analysis. Am J Respir Crit Care Med. 2017 May;195:A1032.

15 Wen XH, Li Y, Han D, Sun L, Ren PX, Ren D. The relationship between cognitive function and arterial partial pressure 02 in patients with COPD: A meta-analysis. Medicine (Baltimore). 2018 Jan;97(4):e9599.

16 Lahousse L, Tiemeier H, Ikram MA, Brusselle GG. Chronic obstructive pulmonary disease and cerebrovascular disease: A comprehensive review. Respir Med. 2015 Nov;109(11):1371-80.

17 Yohannes AM, Chen W, Moga AM, Leroi I, Connolly MJ. Cognitive impairment in chronic obstructive pulmonary disease and chronic heart failure: a systematic review and meta-analysis of observational studies. J Am Med Dir Assoc. 2017 May;18(5):451.e1-11.

18 Baird C, Lovell J, Johnson M, Shiell K, Ibrahim JE. The impact of cognitive impairment on self-management in chronic obstructive pulmonary disease: A systematic review. Respir Med. 2017 Aug;129:130-9.

19 Torres-Sánchez I, Rodríguez-Alzueta E, Cabrera-Martos I, López-Torres I, Moreno-Ramírez MP, Valenza MC. Cognitive impairment in COPD: a systematic review. J Bras Pneumol. 2015 Mar-Apr;41(2):182-90.

20 Shang Y. The risk factors of Alzheimer's disease among Tibetan aged 60 years and older in Qinghai Province. Guangzhou: Southern Medical University; 2015.

21 Frohnhofen H, Heuer HC, Willschrei HP, Falkenhahn C. Dementia among older in-hospital patients with obstructive airway disease: frequency and consequences. Z Gerontol Geriatr. 2011 Aug;44(4):240-4.

22 Kakkera K, Padala KP, Kodali M, Padala PR. Association of chronic obstructive pulmonary disease with mild cognitive impairment and dementia. Curr Opin Pulm Med. 2018 Mar;24(2):173-8.

23 Mahan RJ, Blaszczyk AT. COPD exacerbation and cholinesterase therapy in dementia patients. Consult Pharm. 2016 Apr;31(4):221-5. 
24 Muo I, Malhotra K, Mesioye A. COPD and newly diagnosed dementia in a female elderly patient: is there a possible link? J Am Med Dir Assoc. 2014 Mar;15(3):B5-5.

25 Yeh JJ, Wei YF, Lin CL, Hsu WH. Effect of the asthma-chronic obstructive pulmonary disease syndrome on the stroke, Parkinson's disease, and dementia: a national cohort study. Oncotarget. 2017 Dec;9(15):12418-31.

26 Liao KM, Ho CH, Ko SC, Li CY. Increased risk of dementia in patients with chronic obstructive pulmonary disease. Medicine (Baltimore). 2015 Jun; 94(23):e930.

27 Liao WC, Lin CL, Chang SN, Tu CY, Kao CH. Reply: The association between chronic obstructive pulmonary disease and dementia: a population-based retrospective cohort study. Eur J Neurol. 2015 Jun;22(6):e71.

28 Chang Q, He Y, Wang LN, Jiang Y, Shi GS. A case-control study on COPD and Alzheimer's Disease in elderly military male. Chin J Dis Contr Prevent. 2007 Feb;11(1):37-9.

29 Pietras T, Ciebiada M, Górski P. Dementia and mild cognitive impairment in chronic obstructive pulmonary disease. Psychiatr Pol. 2004;13(1):17-26.

30 Barnes PJ. The cytokine network in asthma and chronic obstructive pulmonary disease. J Clin Invest. 2008 Nov;118(11):3546-56.

31 Fix AJ, Golden CJ, Daughton D, Kass I, Bell CW. Neuropsychological deficits among patients with chronic obstructive pulmonary disease. Int J Neurosci. 1982 Mar;16(2):99-105.

32 Gibson GE, Pulsinelli W, Blass JP, Duffy TE. Brain dysfunction in mild to moderate hypoxia. Am J Med. 1981 Jun;70(6):1247-54.

33 Swardfager W, Lanctôt K, Rothenburg L, Wong A, Cappell J, Herrmann N. A meta-analysis of cytokines in Alzheimer's disease. Biol Psychiatry. 2010 Nov;68(10):930-41.

34 Incalzi RA, Gemma A, Marra C, Muzzolon R, Capparella O, Carbonin P. Chronic obstructive pulmonary disease. An original model of cognitive decline. Am Rev Respir Dis. 1993 Aug;148(2):418-24.

35 Ortapamuk H, Naldoken S. Brain perfusion abnormalities in chronic obstructive pulmonary disease: comparison with cognitive impairment. Ann Nucl Med. 2006 Feb;20(2):99-106.

36 de la Torre JC. Critical threshold cerebral hypoperfusion causes Alzheimer's disease? Acta Neuropathol. 1999 Jul; 98(1):1-8. 